\title{
Factores de estrés en estudiantes universitarios en época de pandemia (Covid-19)
}

\author{
Stress factors in university students in times of pandemic (Covid-19)
}

Fatores de estresse em estudantes universitários em tempos de pandemia (Covid-19)

Jorge Patricio Chávez Reinoso ${ }^{1}$

chavezj_ites@yahoo.es

itscarloscisneros@hotmail.com

https://orcid.org/0000-0002-4079-5050

Dayana Cristina Villarreal Meza
vmdcristina@gmail.com
https://orcid.org/0000-0002-6971-6950
Jorge Ernesto Chávez Guevara²

jorgechavezguevara94@gmail.com jechavez@institutos.gob.ec https://orcid.org/0000-0002-5627-4321

Luis Antonio Ortiz Parra ${ }^{1}$

1.parra4709@gmail.com

https://orcid.org/0000-0003-1116-3545

${ }^{1}$ Instituto Superior Tecnológico Carlos Cisneros, Riobamba-Ecuador

${ }^{2}$ Instituto Superior Tecnológico Juan de Velasco, Riobamaba-Ecuador

Artículo recibido en junio 2021, revisado en julio 2021, arbitrado en agosto 2021 y publicado en septiembre 2021

\section{RESUMEN}

El presente artículo tiene como propósito dar a conocer los factores determinantes del estrés en los estudiantes universitarios y analizar como este fenómeno tiene influencia en el desempeño académico de enseñanza y aprendizaje. El estudio fue ejecutado en época de pandemia (mayo 2021), realizado con enfoque mixto, siendo un estudio descriptivo, transversal, de campo, documental con el apoyo bibliográfico; el cual permitió identificar los factores de estrés y como estos afectan al proceso de enseñanza y aprendizaje en estudiantes de las diferentes facultades de la Universidad Nacional de Chimborazo, en sus diversas carreras y especialidades. De acuerdo con los resultados alcanzados, el 57\% manifiesta que la metodología de educación virtual ha sido muy y extremadamente estresante, al 70\% de los estudiantes les ha afectado la educación virtual y se encuentran estresados, el $72 \%$ desea regresar a la educación presencial, el $88 \%$ afirman que las tareas han generado mayor estrés, el $86 \%$ indica que la pandemia le ha afectado su rendimiento académico, y finalmente que la situación económica y los problemas familiares son factores que han generado mayores niveles de estrés en los alumnos. Se concluye por tanto que la educación virtual ha provocado en los estudiantes de educación superior afectación en niveles de estrés durante la pandemia, impactando en el ámbito psicológico, conductual y cognitivo.

Palabras clave: Pandemia; Covid-19; Educación virtual; Stres; Estudiantes universitarios

\section{ABSTRACT}

The purpose of the present article is to make known the determining factors of stress in university students and to analyze how this phenomenon influences academic performance in teaching and learning. The study was carried out during the pandemic period (May 2021), with a mixed approach, being a descriptive, cross-sectional, field, documentary study with bibliographic support; which allowed identifying the stress factors and how they affect the teaching and learning process in students of the different faculties of the National University of Chimborazo, in their various careers and specialties. According to the results, $57 \%$ state that the virtual education methodology has been very and extremely stressful, $70 \%$ of the students have been affected by virtual education and are stressed, $72 \%$ wish to return to faceto-face education, $88 \%$ state that homework has generated greater stress, $86 \%$ indicate that the pandemic has affected their academic performance, and finally that the economic situation and family problems are factors that have generated higher levels of stress in students. It is therefore concluded that virtual education has affected stress levels in higher education students during the pandemic, impacting the psychological, behavioral and cognitive areas.

Key words: Pandemic; Covid-19; Virtual education; Stres; University students

\section{RESUMO}

O objetivo do presente artigo é identificar os determinantes do estresse em estudantes universitários e analisar como este fenômeno influencia o desempenho acadêmico no ensino e na aprendizagem. O estudo foi realizado durante o período da pandemia (maio de 2021), com uma abordagem mista, sendo um estudo descritivo, transversal, de campo e documental com apoio bibliográfico; o que nos permitiu identificar os fatores de estresse e como eles afetam o processo de ensino e aprendizagem dos estudantes das diferentes faculdades da Universidade Nacional de Chimborazo, em suas diversas carreiras e especialidades. De acordo com os resultados, 57\% afirmaram que a metodologia da educação virtual tem sido muito e extremamente estressante, $70 \%$ dos estudantes foram afetados pela educação virtual e estão estressados, $72 \%$ desejam voltar à educação presencial, $88 \%$ afirmam que o dever de casa gerou maior estresse, $86 \%$ indicam que a pandemia afetou seu desempenho acadêmico e, finalmente, que a situação econômica e os problemas familiares são fatores que geraram maiores níveis de estresse nos estudantes. Conclui-se, portanto, que a educação virtual afetou os níveis de estresse dos estudantes do ensino superior durante a pandemia, com impacto nas esferas psicológica, comportamental e cognitiva.

Palavras-chave: Pandemia; Covid-19; Educação virtual; Stres; Estudantes universitários. 


\section{INTRODUCCIÓN}

La pandemia por efecto del coronavirus o Covid-19 hasta la actualidad ha cambiado las condiciones del sistema educativo en todo el mundo, obligando a las instituciones de educación superior a utilizar la modalidad virtual online, lo cual ha generado varios problemas en los procesos académicos de los estudiantes no solo en la enseñanza y aprendizaje, sino también en lo económico y principalmente en los niveles de estrés, ansiedad, depresión, preocupación, afectando los niveles de conocimiento y fundamentalmente en la salud mental de los educandos.

Bajo estos argumentos, Gutiérrez (2016) indica que la educación virtual simboliza la emergencia del nuevo sistema educativo en el cual existe un respaldo pedagógico con cartas conceptuales y mapas mentales innovadores, debido a que mediante él se puede establecer el encuentro comunicativo entre los actores del proceso. Adicionalmente, Expósito y Marsollier (2020) manifiesta que los sistemas educativos tienen un reto durante los últimos meses relacionado con mantener la vitalidad de la educación y promover el desarrollo del aprendizaje significativo. Para ello, ha contado con dos aliados clave uno de ellos es el docente y el segundo la virtualidad, en términos más precisos, los docentes a través de la virtualidad.

En este contexto y como lo confirman BravoGarcía y Magis-Rodríguez (2020) la modalidad virtual ha sido la principal herramienta en la educación, debido a la importancia que ha adquirido la educación online durante el periodo del 2020 y 2021, producto de un hecho sin precedentes que marca un antes y un después dentro de las prácticas pedagógicas y en los sistemas educativos actuales a nivel global. Asimismo, se ha puesto en evidencia las desigualdades sociales, culturales y económicas de más de 180 países que han sido víctimas de la pandemia por COVID-19.
Sobre la modalidad virtual, el Banco Interamericano de Desarrollo (BID, 2020), plantea que la modalidad de enseñanza remota está siendo creada e implementada de forma abrupta debido a la velocidad que llevan a cabo el proceso, esto marca un hecho sin precedente, y se caracteriza por contar con una combinación de soluciones y medios de primera generación (materiales impresos, radio y televisión) y de segunda generación (plataformas, sistemas de gestión de aprendizajes) para entregar contenido y mantener algún nivel de interacción entre escuelas y estudiantes.

Reforzando esta temática y de acuerdo con Velásquez (2020) que manifiesta que la tutoría virtual se construye mediante el proceso de acompañamiento dentro del aprendizaje, mencionando que el éxito radica en una combinación de metodologías para fortalecer el desarrollo en la educación. Además, indica que no todos los docentes tienen a bien utilizar los entornos virtuales, debido a la contingencia sanitaria en la que se encuentra la humanidad, esto conlleva a una necesidad de aplicar la virtualidad, por lo tanto, se busca mediante la aplicación de teoría y conceptos básicos determinary analizar las funciones y roles del tutor virtual, así como identificar las competencias idóneas que el tutor virtual debe poseer (p. 20).

Anexo, como la manifiesta en su estudio Marciniak, y Gairín-Sallán (2018) para que una modalidad de educación virtual sea de calidad, debe contemplar ciertos requisitos, además, se puede contar con los recursos tecnológicos adecuados y el servicio necesario para acceder al programa educativo; que la estructura y el contenido del curso virtual ofrezcan un valor formativo; que se realicen aprendizajes efectivos y que sea un ambiente satisfactorio tanto para los estudiantes como para los profesores. 
Sin embargo, de contar con una herramienta fundamental que es la virtualidad en la educación en general y en particular en la educación superior, esta forma de enseñanza y aprendizaje ha afectado grandemente al sector estudiantil, en diferentes factores, como el proceso académico, económico, de salud, y fundamentalmente en su salud mental, generando niveles de estrés, que afectan en gran medida su rendimiento estudiantil.

Por efecto de la pandemia, hoy en día se tiene una vida muy acelerada, existe una nueva normalidad en todas las actividades cotidianas de las personas, no hay tiempo para hacer todo lo que se tiene previsto o planificado efectuar, de estas presiones y aceleraciones de vida no están excepto los estudiantes universitarios. El estudiante universitario tiene que lidiar constantemente con la elaboración de trabajos e informes, presentación de proyectos, exámenes, reuniones, eventos, entre otros. Y al tener una sobrecarga de todos estos sucesos se presenta el estrés el mismo que ha traído consecuencias y repercusiones en ámbitos como el académico, psicológico e incluso físico.

Según Usuriaga, (2018) en relación al estrés manifiesta que este surge a raíz del cansancio cognitivo o mental, que se da luego dehaber sometido al sujeto a acciones que superan su rendimiento habitual sin proporcionarle un descanso previo. (p. 25). Siguiendo este mismo tema Escobar, et al., (2018) manifiesta que la incorporación a la universidad constituye una experiencia estresante que da para afrontar situaciones que buscan cambiar la forma de enfocar el aprendizaje, el estudio y la esfera personal, dichos cambios aumentan el riesgo de que los estudiantes presenten altos niveles de estrés.

Por otro lado, el estrés es un término algo complejo e interesante del cual se habla mucho en la actualidad, y que no tiene un consenso en su definición; en si es una respuesta del cuerpo entre una exigencia o presión y la capacidad que se tiene para enfrentarla, provocando desequilibrio en los individuos que lo padezcan. El estrés es el conjunto de procesos y respuestas neuroendocrinas, inmunológicas, emocionales y conductuales ante situaciones que significan una demanda de adaptación mayor que lo habitual para el organismo, y/o son percibidas por el individuo como amenaza o peligro, ya sea para su integridad biológica o psicológica.

Se entiende al estrés como un proceso de cambios que se da en el transcurso de vida del ser humano en el desarrollo del aprendizaje, que por efecto de estar sujeto a riesgos en sus actividades ya sean académicas, familiares, laborales o personales ha conllevado a que los estudiantes no han podido solucionarlos de la manera que espera que sucedan las cosas, esto ha ocasionado cansancio que ha intervenido en su salud física y mental, lo cual afectó su rendimiento académico y estudiantil. Por ello, y como lo confirma en su investigación Albán y Calero, (2017) "el rendimiento académico puede ser entendido en relación a un grupo social que fija los niveles mínimos de aprobación y máximos de desaprobación ante un determinado cúmulo de conocimientos y/o aptitudes". (p. 214).

El estrés ha tenido según el estudio una gran influencia en el rendimiento académico de los alumnos universitarios, los cuales pueden considerarse inconvenientes, obstáculos o problemas que están bajo su responsabilidad, es decir, todas las actividades estudiantiles para el logro de sus objetivos y metas. Dentro de estas actividades las más comunes y generadoras de estrés en los estudiantes universitarios son las evaluaciones académicas, los docentes, las exposiciones, la acumulación de tareas, los procedimientos de aprendizaje, etc. Como consecuencia de esto los 
estudiantes tienen un bajo rendimiento, afectando en su salud física y mental.

En cuanto al estrés académico el estudiante puede percibirlo de dos maneras la primera negativa, que se refiere a situaciones difíciles y angustiantes en el camino de su formación universitaria, siendo estas complejas de resolver llegando a perder el control, entre estas se encuentran: la cantidad de materias, tareas y actividades que deja el docente, la necesidad de enfocar su concentración y atención, la evaluación del profesor en cuanto a su rendimiento académico y comportamiento y la cantidad de estudiantes en cada aula virtual que puede desfavorecer al aprendizaje más personalizado. Y la segunda positiva en donde el estudiante se motiva a realizar trabajos autónomos para obtener algún beneficio, este influye de manera efectiva en sus emociones.

Bajo la pregunta de investigación se manifiesta si ¿La educación virtual durante la pandemia por efecto del Covid-19 o coronavirus, ha afectado a los estudiantes universitarios en el proceso de enseñanza y aprendizaje su rendimiento académico?

Bajo los argumentos expuestos, el objetivo fundamental de este estudio es conocer desde la figura de los estudiantes de la Universidad Nacional de Chimborazo (UNACH), los niveles y factores de estrés al momento de recibir las clases a través de la modalidad virtual, así como identificar como han sido afectados en sus procesos de enseñanza y aprendizaje, durante los días de pandemia, por efecto de la suspensión de clases en la modalidad presencial, a consecuencia de la pandemia del coronavirus o Covid-19.

\section{MÉTODO}

Se realizó un estudio descriptivo, de corte transversal exploratorio, bajo la modalidad mixta (cuantitativa y cualitativa), utilizando investigación de campo con la aplicación de encuestas; y bajo el respaldo de consultas en revistas científicas acreditadas por diversas plataformas y bases de datos. El estudio fue realizado en mayo de 2021, durante la época de pandemia. De acuerdo con Flores (2011), la población es "el conjunto de unidades de observación que poseen características específicas determinadas por el investigador según los objetivos que persigue" (p. 203). La población de estudio estuvo compuesta por los estudiantes de las diversas facultades de la Universidad Nacional de Chimborazo (UNACH), que estuvieron cursando el periodo académico marzo-septiembre 2021; como se observa en la Tabla 1.

Tabla 1. Población y muestra del estudio.

\begin{tabular}{lccc}
\hline \multicolumn{1}{c}{ Facultad } & Población total & \% de la población & $\begin{array}{c}\text { Encuestas } \\
\text { aplicadas }\end{array}$ \\
\hline Ciencias de la Educación, Humanas y Tecnológicas & 1.675 & 19,12 & 70 \\
Ciencias de la Salud & 2.816 & 32,13 & 118 \\
Ciencias Políticas y Administrativas & 2.109 & 24,06 & 89 \\
Ingenierías & 2.164 & 24,69 & 91 \\
TOTAL & $\mathbf{8 . 7 6 4}$ & $\mathbf{1 0 0 , 0 0}$ & $\mathbf{3 6 8}$ \\
\hline
\end{tabular}

Fuente: datos facilitados por la Unidad Técnica de Control Académico UNACH, 2021. 
Para calcular la muestra se utilizó la fórmula de Vara (2012, p. 227), que se presenta a continuación:

$$
n=\frac{Z^{2} p \cdot q \cdot N}{e^{2}(N-1)+Z^{2} p \cdot q}
$$

$$
\mathrm{n}=\frac{\left(\left((1,96)^{2} * 0,5 * 0,5\right)\right) *(8764)}{\left((8764-1) *(0,05)^{2}\right)+(1,96)^{2} * 0.5 * 0.5}
$$

$\mathrm{n}=368$ alumnos encuestados

En donde:

$\mathrm{n}=\quad$ Tamaño de la muestra calculada

$\mathrm{N}=$ Total del universo (8764 estudiantes)

$\mathrm{Z}=\quad$ Nivel de confianza (95\%, equivalente a 1,96 en la tabla de Gauss)

$\mathrm{e}=\quad$ Porcentaje de error (5\%)

$\mathrm{p}=$ Ocurrencia de un evento (Probabilidad $50 \%)$

$\mathrm{q}=\quad$ No ocurrencia de un evento (Probabilidad $50 \%)$

\section{Instrumentos y procedimiento de recopilación de información}

Una encuesta fue la principal herramienta para recopilar los datos de fuentes primarias, aplicada a 368 estudiantes que cursaban en la Universidad Nacional de Chimborazo, durante el periodo académico marzo-septiembre del 2021, para ello, se usó el método de muestreo probabilístico estratificado proporcional. La escala de Likert (1932) se utilizó para la recopilación de la información, de acuerdo con (Bertram, 2008) y citado por Matas (2018), es "Un instrumento psicométrico donde el encuestado debe indicar si está de acuerdo o desacuerdo sobre una afirmación, ítem o reactivo, lo cual se realiza a través de una escala ordenada y unidimensional" (p. 39).

\section{Procesamiento de datos}

Una vez recopilados los datos se utilizó el programa estadístico Statistical Packge for Social Sciences (SPSS), para tabular la información, y su posterior análisis e interpretación.

\section{RESULTADOS Y DISCUSIÓN}

\section{Análisis del nivel de estrés en estudiantes universitarios en época de pandemia por efecto de la educación virtual}

La relación personales entre compañeros de clase es importante en los procesos académicos, de acuerdo a los datos obtenidos apenas el 30\% de los estudiantes se encontraba muy estresados y extremadamente estresados por la educación virtual y la pandemia con respecto a este nuevo tipo de relación interpersonal con sus compañeros por un medio digital, mientras que el $32 \%$ de estudiantes se encuentra moderadamente estresados, siendo esta evidencia que se podría mencionar como un estrés normal manejado por los estudiantes, mientras que el $28 \%$ están algo estresados, lo que permitió observar que los estudiantes manejaban un estrés un tanto más bajo de lo normal aunque este fenómeno se mantiene en los estudiantes no de una manera alarmante pero se nota que si manejan cierto grado de estrés.

Seguidamente, en relación a la modalidad de estudio, esto es virtual o en línea la relación entre docentes y estudiantes se vuelve diferente aun cuando se está en clases de manera presencial, por lo que se evidencia que se encuentra que en un porcentaje más alto de las opciones presentadas muy estresante con una tendencia hacia extremadamente estresante, con un $57 \%$.

En cuanto a la percepción de las relaciones entre docentes y estudiantes, la comunicación entre docentes y estudiantes se hace más difícil, así como 
la praxis aplicada puede ser el hecho que detone el estrés en los estudiantes puesto que en el $32 \%$ de los estudiantes encuestados se perciben muy estresados. Luego se evidenció que los estudiantes encuestados perciben su experiencia de haber tomado estudios académicos en épocas de pandemia de Covid-19 con presencia de estrés, donde el $40 \%$ de estudiantes que dicha experiencia ha sido moderadamente estresante, el $28 \%$ perciben dicha experiencia académica como muy estresante, el $17 \%$ mencionan que ha sido extremadamente estresante cursar sus estudios en épocas de pandemia y el 15\% restante menciona que ha sido algo estresando haber tenido dicha experiencia académica. Así también, se precisa mencionar que no ha habido estudiantes que aseveren el ausentismo de estrés en cuanto a su percepción de la experiencia de haber cursado estudios académicos en épocas de Covid-19. (Figura $1)$.

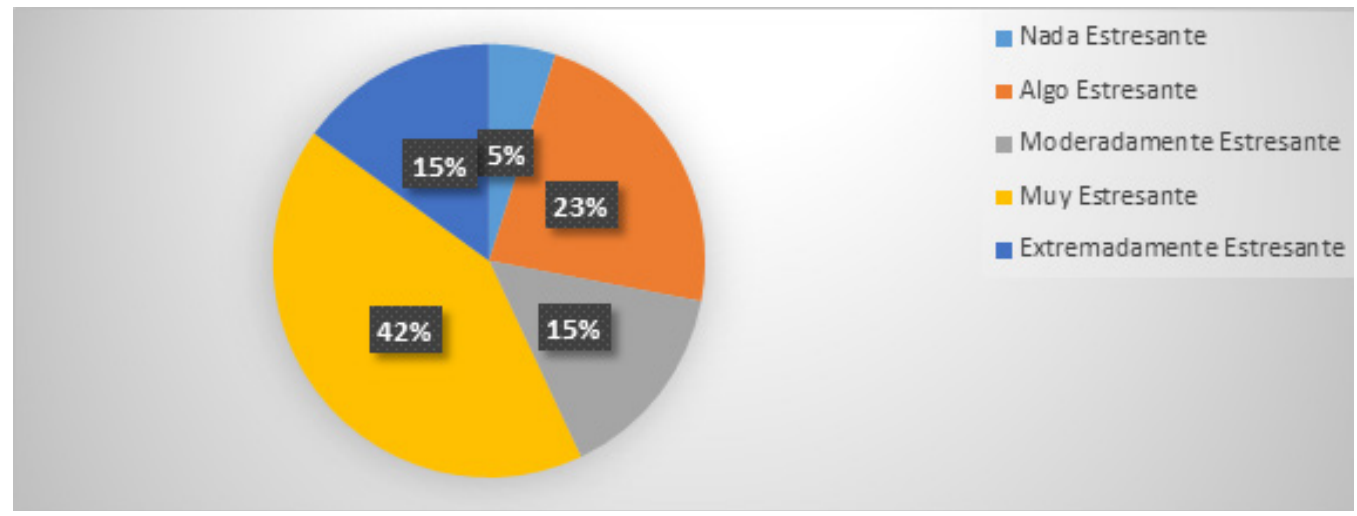

Figura 1. Experiencia del estudio académico en pandemia.

Las tareas académicas universitarias en educación han sido en su mayoría bajo la modalidad virtual y respecto a la forma en la que perciben la metodología de educación virtual los estudiantes encuestados, se pudo evidenciar que el $42 \%$ mencionaron que ello ha sido muy estresante, el $15 \%$ indican que esto ha sido extremadamente estresante, en conjunto suma un 57\%, esto significa que casi 3 cuartas partes consideraron que la educación virtual si ha afectado en los niveles de estrés durante este período de la pandemia de COVID-19. (Figura 2).

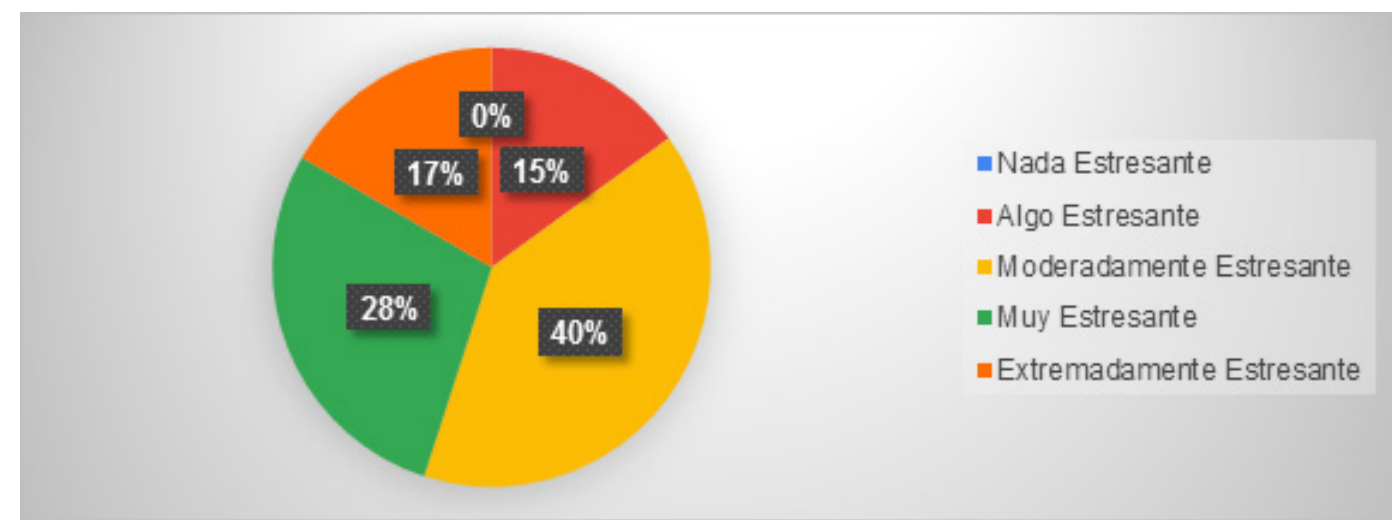

Figura 2. Percepción de la metodología de educación virtual durante la pandemia. 
Los dispositivos electrónicos (Computador personal, laptop, celular y Tablet), han sido los más utilizados en esta época de educación virtual, el 39\% y $21 \%$ de los estudiantes consideran muy estresante y extremadamente estresante respectivamente el uso de los dispositivos electrónicos, un 31\% indican como algo y nada estresante. Esto obedece a que los dispositivos electrónicos fueron las herramientas más utilizadas; lo cual permite concluir que se debe tomar en cuenta que el tiempo frente al computador durante el periodo de pandemia es elevado, genera mucho estrés e incluso afecta la visión de las personas por el alto uso.
De conformidad al sistema de educación virtual, los docentes han enviado muchas tareas y actividades a sus estudiantes, sin embargo esto no ha ocasionado que los niveles de estrés sean altos, se puede observar que el $38 \%$ indica que es algo estresante, mientras que un $22 \%$ moderadamente estresante, apenas un 10\% manifiesta que la carga de trabajos es muy estresante, lo cual permite concluir que al existir sobre carga de tareas académicas los estudiantes se tienden a sentir en su mayoría algo estresantes. Pero las asignaciones de tareas manejan niveles aceptables de estrés en pandemia. (Figura 3).

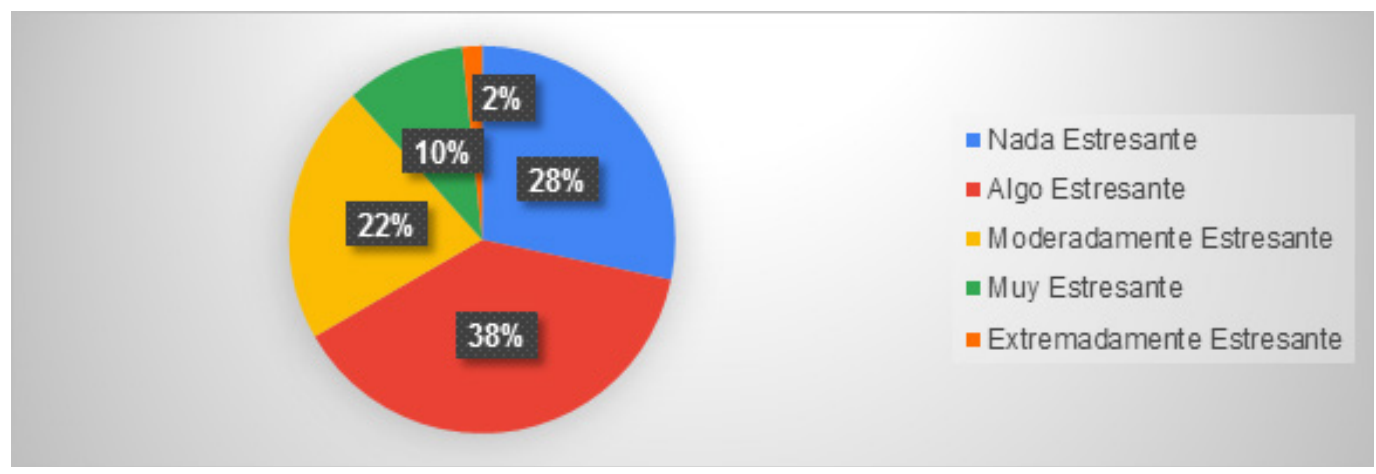

Figura 3. Sobrecarga de tareas universitarias.

La educación virtual como una modalidad de estudio ha sido utilizado por todo el mundo en el ámbito educativo, a través del uso del dictado de clases, envió de tareas, presentación de actividades y proyectos, mediante la asignación de tareas sincrónicas y asincrónicas, esto ha provocado ciertos síntomas de estrés en los estudiantes universitarios, en donde se determinó que el 70\% de los consultados se sienten estresados en general, apenas un $12 \%$ manifiesta no haber tenido síntomas de estrés.

Se desprendió que el $36 \%$ de los encuestados se siente conformes con la modalidad de estudio virtual, seguido por $28 \%$ que afirma que se siente muy conforme, $18 \%$ se sienten poco conforme,
$10 \%$ nada conforme y finalmente $8 \%$ se sienten demasiado conforme. Esto obedece a que el tema de los contagios si las clases se realizan de manera presencial se generan muchos más problemas en los estudiantes, por efecto de las consecuencias que se pueden presentar.

La pandemia por el aparecimiento del virus ha generado muchos inconvenientes en los económico, social, productivo, en las actividades turísticas y principalmente en lo educativo, lo cual ha generado que los estudiantes universitarios tengan el deseo de regresar a clases de manera presencia, por ello en cuanto a esta pregunta podemos observar que el $72 \%$ de los encuestados quisieran volver a clases presenciales y el $28 \%$ no quiere volver a clases 
presenciales. Esto evidencia las dificultades en todos los ámbitos que ha generado la pandemia por el Covid-19.

Para que exista un buen desempeño académico en los procesos de enseñanza y aprendizaje en los estudiantes universitarios, deben garantizarse las condiciones adecuadas para lograr este objetivo, sin embargo, el tema de la pandemia por el miedo al contagio ha provocado muchos problemas y con respecto a esto se evidencia que el $86 \%$ de los encuestados sienten que la modalidad de estudio virtual ha afectado a su desempeño académico y tan solo el $14 \%$ siente que esta modalidad de estudio no ha afectado a su desempeño académico. Se considera por tanto un nivel alto las consecuencias de la modalidad virtual. (Figura 4).

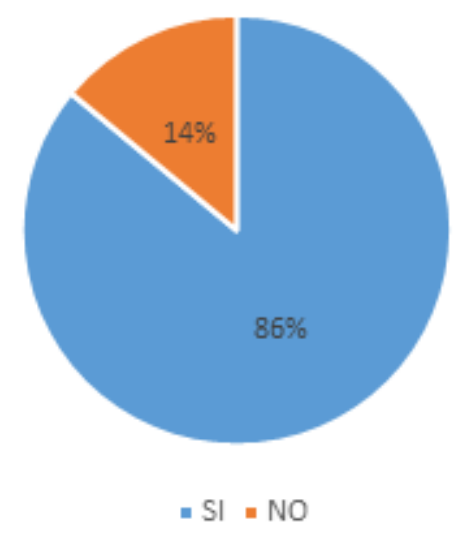

Figura 4. Desempeño de los estudiantes universitarios por la pandemia.

Complementado con lo descrito en el tema anterior sobre el desempeño académico de los estudiantes, se puede evidencia que de acuerdo con el aprendizaje bajo la modalidad virtual los resultados arrojan un empate ya que el $50 \%$ de los encuestados considera que está aprendiendo bajo la modalidad de estudio virtual y el otro $50 \%$ considera que no. Esto permite determinar que el nivel de conocimientos de los estudiantes no es el adecuado en la actualidad. (Figura 5).

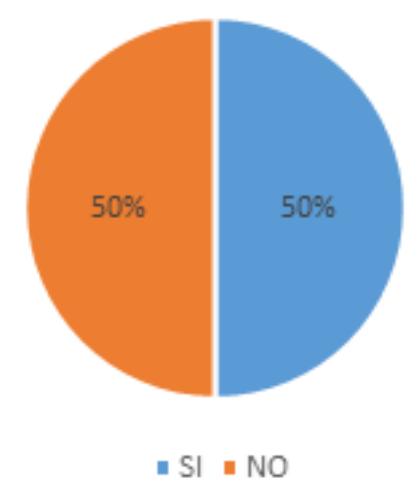

Figura 5. Aprendizaje de los estudiantes universitarios por la pandemia. 
En cuanto a la la causas por estrés (Figura 6) se pudo determinar que la mayoría con un $88 \%$ del total de los encuestados opinaron que la principal causa de estrés es el exceso de tareas y tan solo el $12 \%$ opinaron que el exceso de tareas no es causante de estrés, todo esto en contexto de tiempos de pandemia por Covid-19. Lo que conlleva a pensar que las tareas enviadas por los docentes generan altos niveles de estrés en el sector estudiantil, debiendo los docentes buscar mejores y mayores estrategias para mejorar el proceso de enseñanza y aprendizaje.

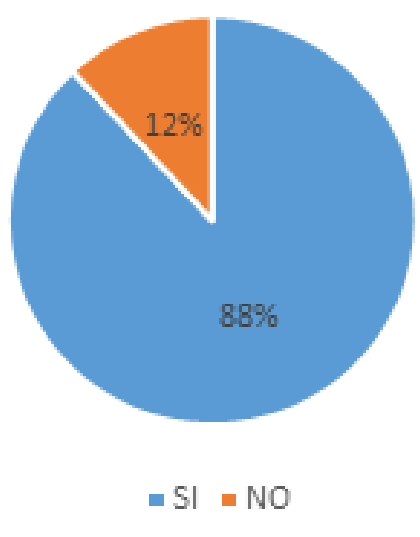

Figura 6. Aprendizaje de los estudiantes universitarios por la pandemia.

La parte psicológica al igual que la parte educativas son factores importantes en el desempeño personal del sector estudiantil, como se muestra en la Figura 7 con relación a este tema, se puede evidencia que los estudiantes han manifestado los siguientes datos: alumnos ansiosos un $28,3 \%$, preocupados $26,7 \%$, desconcentrado un $30 \%$ y desorganizados un $15 \%$. La mayor dificultad aparece en alumnos ente ansioso y desconcentrado con 2 terceras partes del total del alumnado.

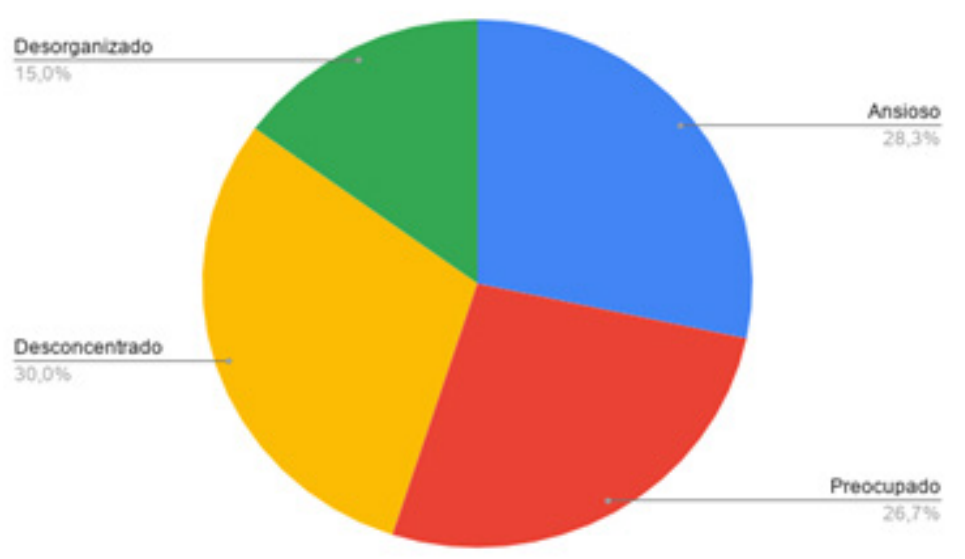

Figura 7. Aspectos personales que han sentido los estudiantes en época de pandemia. 
Existen muchos factores o razones que (38,3\%), seguido de los problemas familiares con el causan preocupación o estrés en los estudiantes universitarios, sin embargo, fruto de esta investigación se ha evidenciado que la situación económica ha generado mayor porcentaje de estrés $26,7 \%$, la situación académica con un $20 \%$, y el medio contagio un $15 \%$. Lo cual en conjunto son factores que de alguna manera causan incertidumbre, miedo y ansiedad en los estudiantes. (Figura 8).

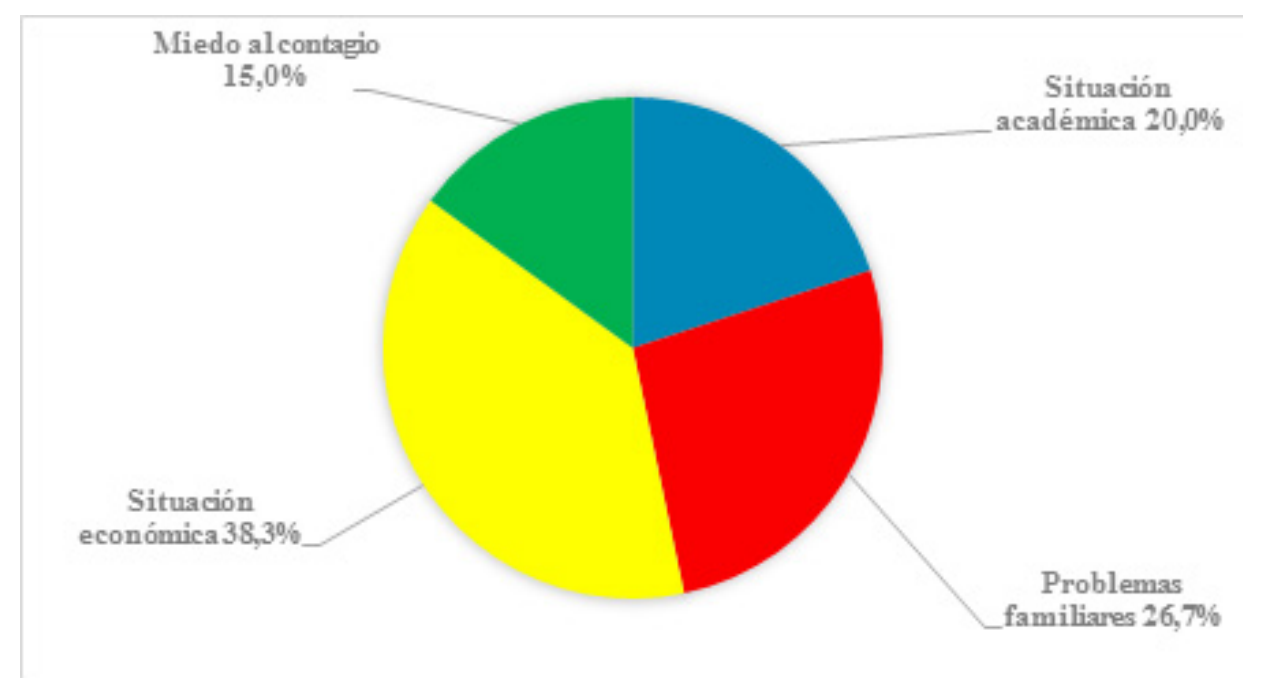

Figura 8. Razones que causan el estrés en estudiantes universitarios en época de pandemia.

El momento de realizar actividades de estudio, esto es tareas, proyectos, actividades, informes por parte del sector estudiantil, se puede demostrar que la falta de explicación del maestro tiene un porcentaje del $41,7 \%$, la falta de contacto del profesor tiene un 21,7\%, la falta de comprensión del maestro de las necesidades del estudiante tiene un $20 \%$, la falta de compresión del estudiante tiene un 16,7\%. (Figura 9).

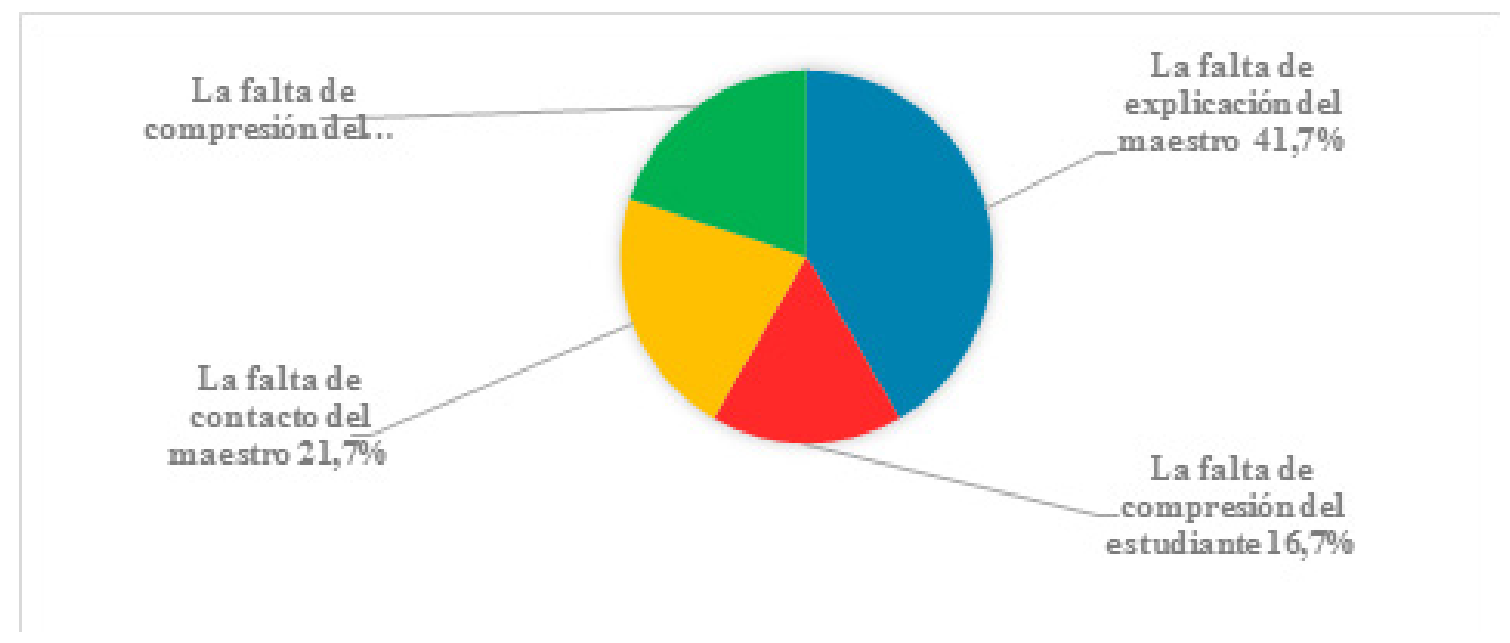

Figura 9. Aspectos por los cuales genera estrés en estudiantes durante el estudio. 
Se puede observar en la Figura 10 que las clases presenciales tienen un porcentaje del $48,3 \%$ es decir los estudiantes desean las clases de manera presencial, las clases virtuales con un 33,3\% y los dos en conjunto el 18,3\%. Demostrando que la modalidad de estudio virtual debe ser suplantada por la presencial, a nivel de estudios universitarios.

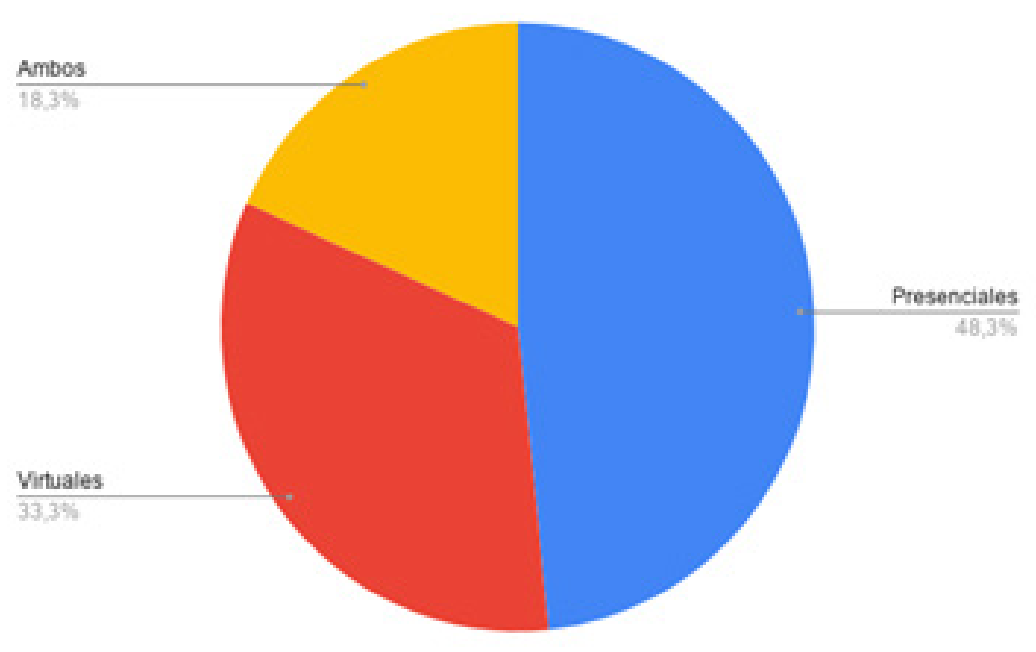

Figura 10. Modalidad de estudio que prefiere el sector estudiantil.

\section{Discusión}

La pandemia por el aparecimiento de la Covid-19 o coronavirus obligó en especial al sistema educativo a nivel superior, al confinamiento y asilamiento, las actividades de enseñanza y aprendizaje se desarrollaron de manera virtual, en donde el docente utilizando medios electrónicos y tratando de idear diferentes estrategias de enseñanza, trató de llegar hacia los educandos y estos a través de varios mecanismos procurar captar los conocimientos de sus docentes.

El estrés en estudiantes universitarios es una variable dependiente que tiene mucha repercusión en el rendimiento académico de los que atravesando por este estado de tensión. Desde esta perspectiva la investigación se centra en el estrés académico, que según García, (2017), indica que "estrés académico se refiere a los procesos cognitivos y afectivos que permiten a los estudiantes percibir el impacto que causan los estresores académicos" (p. 67), y en la actualidad el estrés se ha convertido en una de las causas más importantes de muerte en todo el mundo, y que afecta a la mayoría de las personas indistintamente del sector o la actividad a la que se dediquen, y los estudiantes universitarios no son la excepción. Son varios los factores que conducen a una persona a este estado de tensión, ya sea personales, familiares o en el caso de los estudiantes universitarios por problemas académicos que son los más comunes.

Por ello, como lo manifiesta Hurtado (2020) ante esta nueva realidad a consecuencias del COVID-19, la educación se ha transformado notándose que las escuelas han quedado vacías y las casas se han convertido en los espacios en donde se aprenden valores, y también en los escenarios de nuevos debates de aprendizajes formal e informal, este estudio demuestra los cambios dentro del 
proceso de desarrollo de actividades académicas, específicamente en eso nuevos escenarios los cuales generan estrés dentro del educando.

Todo este contexto de la educación virtual, permite visualizar que como lo indica Menendes (2012), citado por Moreno (2020), la virtualidad suponen un reto para el quehacer docente, también para instituciones educativas, considerando que tanto los docente y los estudiantes se encuentra sumidos con métodos tradicionales en el modelo educativo a través de clases magistrales, en este estudio se pudo determinar que existe un cambio dentro de los indicadores de como actualmente los involucrados en el proceso pedagógico denotan este cambio de presencialidad y virtualidad destacando que la mayoría desean ir a las aulas, pero un considerable porcentaje que manifiesta interés en la educación mixta. Sin embargo, desde que ha sido aplicado este sistema de dictado de clases mediante la modalidad virtual, todo este conjunto de acciones generó niveles de estrés en diferentes ámbitos en los estudiantes universitarios, lo cual ha provocado bajo rendimiento académico, ansiedad, preocupación, problemas en la salud física y mental en los estudiantes.

Siguiendo con este orden, para que pueda existir una complementación eficiente en el proceso educativo, es importante la relación entre compañeros de aula, por cuanto son entre ellos quienes van a compartir las experiencias estudiantiles y de sus docentes; lo que se ha evidenciado niveles de estrés moderados en cuanto a la relación a con sus compañeros de aula. En cuanto a la relación con sus docentes, no ha existido la presencia de estrés, apenas una tercera parte de los alumnos indican la afectación por el estrés en la relación docente-estudiantes.

A nivel educativo en los diferentes niveles, $\mathrm{y}$ fundamentalmente en el universitario, nadie se imaginó la presencia de una pandemia de esa magnitud, que tomó desprovisto de herramientas de trabajo estudiantil a los estudiantes y docentes, por lo que se puede manifestar que esta es una experiencia que ha tenido niveles de estrés moderadamente estresante. Adicional, la metodología de educación virtual implementada en todo el sector educativo ha generado niveles de estrés entre muy y extremadamente estresante.

En esta época crítica el uso de equipos tecnológicos y al sistema de internet han sido factores importantes al memento de dictar clases los docentes y al recibir por parte de los estudiantes, y a pesar de que el sistema educativo está acostumbrado a operar con diferentes tipos de equipos, sea laptops, computadores personales o tablets, se ha podido evidenciar que existen niveles de estrés en los alumnos en grado entre muy y extremadamente estresante; aquí el uso del internet ha ayudado mucho en los procesos académicos. Concomitante con lo investigado también se puede afirmar que debido al uso el internet se ha congestionado ocasionado preocupación y ansiedad en los estudiantes, como lo afirma Beltrán y Vanegas (2020): (...) hay alrededor de un $20 \%$ de familias que no disponen de ordenador o de conexión a internet. Esta cuestión es imprescindible para poder seguir la enseñanza no presencial. Aparte, aún las familias que tienen, algunas necesitan el ordenador por el teletrabajo de la madre y/o el padre, o bien, algunos progenitores han de desplazarse al lugar de trabajo (...). Esto dificulta el aprendizaje a este alumnado. (p. 101).

El conjunto de acciones referentes al entorno de la educación virtual, indican que existe conformidad en el proceso educativo, tanto de enseñanza como de aprendizaje de los alumnos, los estudiantes han afirmado sentirse conformes y muy conformes con un porcentaje alto, sin embargo de ello manifiestan 
que la educación virtual o en línea ha afectado en gran medida su desempeño académico, lo cual obliga a pensar a las autoridades institucionales de la educación superior en el país a regresar a la modalidad de educación presencial, pues las 2 terceras partes de los alumnos así lo requieren.

Bajo este contexto, El estrés académico es un estado que se produce cuando un estudiante percibe negativamente las demandas del medio (distrés), es decir, que estas situaciones a las que se enfrenta le resultan angustiantes durante su formación académica y pierde el control sobre ellas, en algunas ocasiones se presentan síntomas físicos como: ansiedad, cansancio, insomnio, bajo rendimiento escolar, desinterés de sus estudios, se ausenta de sus clases e incluso abandonan la carrera. Por el contrario, cuando estas demandas son percibidas positivamente (eustrés), el estudiante se motiva a realizar los trabajos académicos, e influye positivamente en sus emociones. (Zarate, Soto, y Quintero, 2017 pp. 95-96). Como se puede apreciar, los hallazgos permiten visualizar que el estrés ha afectado de manera negativa en los estudiantes universitarios, en la parte académica y obviamente esto repercute en los aspectos personales; así se nota a los estudiantes en época de pandemia desorganizados en su trabajo, con niveles de ansiedad preocupantes, desconcentrados en sus tareas, actividades, responsabilidades lo que genera intranquilidad. Esta ansiedad generada por el estrés ha sido provocada por la situación económica que se ha vivido por cuanto las actividades económicas prácticamente sufrieron paralización, lo que provocó problemas familiares y el miedo al contagio; todo en conjunto creó que la situación académica de rendimiento estudiantil desmejore y los estudiantes no logren captar de mejor manera los conocimientos de sus docentes.
Así, según Sánchez, (2018), se puede aludir que "el estrés académico es mayor en estudiantes de educación superior debido a la mayor cantidad de exigencias académicas". (p 28-29). Esto se nota por cuanto en la educación superior los niveles de exigencia son mayores, por la cantidad de actividades, tareas, responsabilidades, presentación de proyectos, informes, efectuar tareas de vinculación con la sociedad, trabajo comunitario, participar con sus docentes en proyectos de investigación, desarrollar sus trabajos de titulación, entre otras tareas que debe asumir el estudiante universitario.

En general, la vida de los alumnos en cualquier parte del mundo está llena de presión, más aún en esta época de pandemia, pero cada alumno tiene una visión diferente de su propia presión, que también depende de otros factores, como el auto concepto académico, es decir, cómo los alumnos se ven a sí mismos y con respecto a los demás. Otros factores socioeconómicos para él también determinan la presión subjetiva de este problema llamado estrés, cada alumno controla o no controla su propia presión.

\section{CONCLUSIONES}

En la actualidad Ecuador se encuentra atravesando la crisis sanitaria más preocupante de la historia, esto es la pandemia por efecto del Covid-19, por lo que ha obligado a que los estudiantes a todo nivel y fundamental al universitario reciban sus clases de manera virtual, en ciertos alumnos estas medidas de seguridad que determino el gobierno les provoco niveles de estrés bastante alarmantes; provocando preocupaciones en lo personal, económico, familiar y académico, lo cual ha impacto en aspectos físicos, emocionales y de salud mental, que los estudiantes tuvieron que pasar en época de pandemia. 
A medida que los estudiantes universitarios van interactuando con el medio y consiguiendo sus objetivos o metas, este en el transcurso se encontraron con problemas y obstáculos, los cuales no han podido ser resueltos como se espera esto causa inquietudes, así como, desesperación al no encontrar una solución correcta, por tanto, esto es la generación del estrés, lo cual en la vida cotidiana le afecta, así como en la situación académica, motivo de esta investigación en el ámbito de los procesos de enseñanza y aprendizaje.

Finalmente, se puede afirmar que la vida de los estudiantes universitarios es muy complicada, este es un proceso de estudio a largo plazo y una adaptación constante a diferentes entornos como el caso actual al de la pandemia, solo unos pocos han logrado el propósito de poder culminar los estudios universitarios. Sin embargo, se sabe que estas presiones en el proceso educativo son necesarias y en ocasiones excesivas, pero forman parte de la motivación en la que deben participar los estudiantes a nivel superior; por ello debe existir la dinámica de fortalecer, entrenar y capacitar antes de ingresar a un mercado laboral más competitivo y estresante, como el actual, compuesto por altas dosis de competitividad laboral.

\section{REFERENCIAS}

Albán, J., y Calero, J. L. (2017). El Rendimiento Académico: Aproximación Necesaria a un Problema. Revista Conrado, 213-220

Beltrán, J., y Vanegas, M. (2020). Educar en época de confinamiento: La tarea de renovar un mundo común. Revista de Sociología de la Educación. Vol. 13, núm. 2. Especial Covid-19, pp. 92-104

Bravo-García, E., y Magis-Rodríguez, C. (2020). La respuesta mundial a la epidemia del COVID-19: los primeros tres meses. Boletín sobre COVID-19 Salud Pública y Epidemiología, Vol.
1 número 1, pp. 3-8. http://dsp.facmed.unam. $\mathrm{mx} /$ wp-content/uploads/2013/12/COVID-19No.1-03-La-respuesta-mundial-a-la-epidemiadel-COVID-19-los-primeros-tres-meses.pdf

Escobar, E., Soria de Mesa, B., López, G., y Peñafiel, D. (2018). Manejo del estrés académico; revisión crítica. Revista Atlante

Expósito, C., y Marsollier, R. (2020). Virtualidad y educación en tiempos de COVID-19. Un estudio empírico en Argentina. Revista Educación y Humanismo, 22(39), pp.1-22. DOI: https:// doi.org/10.17081/eduhum.22.39.4214file:///C:/ $\mathrm{U} s$ e r s / G l o b a l O f f i c e / Downloads / 4214 - Texto $\% 20$ de $1 \% 20$ art\%C3\%ADculo-14839-2-10-20200831.pdf

Flores, J. (2011). Construyendo la tesis universitaria. Guía Didáctica. Lima: Garden Graf S.R.L.

Gutiérrez, L. (2016). Deliberación entorno a la Educación Virtual. Interconectando Saberes, vol. (1), pp. 77-89. http://is.uv.mx/index.php/IS/ article/view/1112

Hurtado, F. (2020). La Educación en tiempos de pandemia: Los desafíos de la escuela del siglo XXI, Revista Revencyt del Centro de Investigación y Estudios Gerenciales (CIEG), número 44, julio-agosto 2020, pp. 176-187. ISSN: $2244-8330$

IDB. Inter-american Development Bank. (2020). La educación en tiempos del coronavirus. Los sistemas educativos en América Latina y el Caribe ante COVID19. Disponible en: https:// publications.iadb.org/publications/spanish/ document/La-educacion-en-tiempos-delcoronavirus-Los-sistemas-educativos-deAmerica-Latina-y-el-Caribe-ante-COVID-19. pdf

Marciniak, R.,yGairín-Sallán,J.(2018).Dimensiones de evaluación de calidad de educación virtual: revisión de modelos referentes. RIED. Revista Iberoamericana de Educación a Distancia, 21(1), pp. 217-238. Disponible en: https://doi. org/10.5944/ried.21.1.16182

Matas, A. (2018). Diseño del formato de escalas tipo Likert: Un estado de la cuestión. Revista Electronica de Investigacion Educativa, Vol 20, 
Núm 1, pp. 38-47

Moreno, S. (2020). La innovación educativa en los tiempos del coronavirus. Revista Salutem Scientia Spiritus, 6 (1), pp. 14-26. Recuperado de: https://pesquisa.bvsalud.org/portal/resource/ pt/biblio-1087909

Sánchez, A. (2018). Relación entre estrés académico e Ideación suicida en estudiantes Universitarios. Eureka, 28-29

Usuriaga, M. (2018). Factores de riesgo y la frecuencia de estrés de los estudiantes de odontología. Huánuco: Universidad de Huánuco

Vara-Horna, A. (2012). Desde La Idea hasta la sustentación: Siete pasos para una tesis exitosa. Un método efectivo para las ciencias empresariales. Instituto de Investigación de la Facultad de Ciencias Administrativas y Recursos Humanos. Universidad de San Martín de Porres. Lima

Velásquez, B. (2020). La educación virtual en tiempos de Covid-19. Universidad de San Carlos de Guatemala, 3(1), pp. 19-25. DOI: https://doi. org/10.46734/revcientifica. v2i1.8

Zarate, N., Soto, M., y Quintero, J. (2017). Estrés académico en estudiantes universitarios: medidas preventivas. Revista de la Alta Tecnología y la Sociedad., 92-98 